\title{
Mathematical Simulation of Bioseparation in an Affinity Packed Column
}

\author{
P. Sridhar, N. V.S. Sastri, J.M. Modak and A. K. Mukherjee *
}

\begin{abstract}
Affinity chromatography (biospecific adsorption) relies on specific interactions of biological molecules such as enzymes, antigens, antibodies, and proteins. The process consists of three steps: adsorption, washing, and elution. A mathematical model including convection, diffusion, and reversible reaction is formulated to analyse the breakthrough behaviour of the solute. A moving finite element orthogonal collocation method is applied with respect to the space variables of the governing partial differential equations of the model to evaluate the breakthrough of the solute. Danckwerts' boundary conditions are considered for the column. The validity of the numerical scheme is checked by comparison with an analytical solution for a simplified model. The results obtained from model simulation show that the breakthrough time of the solute is significantly influenced by the axial dispersion coefficient, solute concentration, ligand content, reaction kinetics, particle porosity, particle size, and flow rate. Solute recovery and bed utilisation efficiencies are evaluated for different values of the above parameters.
\end{abstract}

\section{Introduction}

Selective binding capabilities offer advantages over relatively non-specific physical properties when used as the basis of a separation process. Affinity chromatography owes its power as a purification method to specific biological interactions. For example, an enzyme may interact with an immobilised substrate or substrate analogue, an immobilised lectin may interact with the carbohydrate moiety of a glycoprotein or a receptor protein with its specific ligand. The underlying concept of affinity separations is simple: a feed is contacted with a solid phase that has a high affinity for the solute of interest. After the solid phase has been saturated it is washed to remove non-specifically adsorbed contaminants. The solute is collected after disrupting the specific interactions. The high affinity solid phase can be, for example, a solute-specific antibody immobilised on porous particles or, if the solute is an enzyme, an immobilised cofactor or substrate analogue. A large number of affinity systems have been developed for a wide variety of separations $[1-3]$.

Analysis of affinity separations has lagged considerably behind the explosion in its applications. Detailed mathematical analysis for the models has been lacking in the literature. Katoh et al. [4] applied fixed bed adsorption theory with an overall mass transfer coefficient for the numerical prediction of adsorption and elution profiles. They concluded that the performance during adsorption was limited by diffusion rather than by the formation of the affinity complex. Sportsman and Wilson [5], however, modelled the nonequilibrium interactions with a flow rate-dependent rate constant for the formation of the affinity complex. Chase [6] has presented and used a simple model in order to study

\footnotetext{
* P. Sridhar, N. V.S. Sastri (deceased), J. M. Modak and Prof. A.K. Mukherjee (to whom all correspondence should be addressed), Department of Chemical Engineering, Indian Institute of Science, Bangalore 560 012, India.
}

the dynamic behaviour of biospecific adsorption in a fixed bed. The rate constants in Chase's model are parameters which lump the effects of the interaction mechanism between the solute and the ligand together with the mass transfer mechanisms characterising the transport of solutes, since the model does not account for mass transfer resistances. Arnold and Blanch [7] indicate that the equations reported by Chase are not quite correct and Chase's definition of time is ambiguous since it depends on the size of the nonadsorbing species. Arnold et al. [8] presented a model for biospecific adsorption in a fixed bed that includes axial dispersion, liquid film mass-transfer resistance, and diffusion within the adsorbent particles. The Langmuir isotherm was considered, and the rate laws of formation of the affinity complex were first order reversible and second order irreversible. Solutions to the model were obtained by using the approach of Vermeulen et al. [9] which is valid for a plug flow system exhibiting constant pattern behaviour in which the adsorption is irreversible and the rate of the adsorption is very fast. There is evidence that the rate of adsorption step may be rate limiting, and Arve and Liapis [10] found for the experimental system of their study that the finite rates of the association and dissociation steps contributed significantly to the dynamic behaviour of the system. Their results indicate that the assumption of a very fast adsorption step, as assumed in the work of Arnold et al. [8], may not be appropriate in certain biospecific adsorption systems. Modelling of affinity separation on gel beads in a packed bed is presented by Horstmann and Chase [11] without axial dispersion. In a recent paper, Gonzalez-Patino et al. [12], studied the effect of particle size on adsorption equilibrium and mass transfer kinetics with respect to affinity chromatography. They concluded that small particles (55-56 $\mu \mathrm{m}$ diameter) should be used for improving separation efficiency rather than larger particles in order to avoid possible internal resistances. Boyer and Hsu [13] studied the effect of ligand concentration on protein adsorption in dye ligand adsorbents. They included Langmuir's isotherm and an analytical solution was given. Table 1 summarises some 
Table 1. Comparison of mathematical models for affinity chromatography

\begin{tabular}{|c|c|c|c|c|c|c|c|}
\hline Katoh et al. (1978) & Freundlich & yes & yes & no & no & - & HETP \\
\hline Arve and Liapis (1987) & - & yes & yes & yes & yes & $\begin{array}{l}\text { orthogonal } \\
\text { collocation }\end{array}$ & $\begin{array}{l}\text { multi- } \\
\text { component }\end{array}$ \\
\hline Boyer and Hsu (1992) & Langmuir & yes & yes & no & no & analytical & experiments \\
\hline This work & $\begin{array}{l}\text { linear } \\
\text { Langmuir }\end{array}$ & yes & yes & yes & yes & $\begin{array}{l}\text { OC on } \\
\text { a } \mathrm{MFE}^{\mathrm{a})}\end{array}$ & simulation \\
\hline
\end{tabular}

a) Orthogonal collocation on a moving finite element.

existing mathematical models found in the literature for affinity chromatography.

In preparative- and large-scale affinity chromatography, the column is often overloaded in terms of feed volume or concentrations, or both. Thus, interference effects, axial dispersion and mass transfer resistances such as interfacial mass transfer and intraparticle diffusion become important. Mathematical modelling and theoretical analysis play an important role in the scale-up process. The majority of the existing theoretical models for affinity chromatography are designed in a nonlinear concentration range with no mass transfer or kinetic resistances. Most scale-up processes for protein purification using affinity chromatography were carried out empirically. Furthermore, mass transfer resistances can be very significant, especially for macromolecules.

Process modelling and simulation are conducted to gain a better understanding of the solute breakthrough behaviour in packed columns. The objective of the present study is to develop a mathematical model that describes the adsorption stage of affinity chromatography in a packed column from which the breakthrough curves can be generated. This comprehensive model includes axial dispersion, surface film resistance, porous diffusion resistance, and surface reaction kinetics. The solution of this comprehensive model requires numerical computation. A moving finite element orthogonal collocation method is used. The detailed mathematics involved is presented in $[14,15]$. This work focusses on simulating the effect of axial dispersion coefficient, solute concentration, ligand content, reaction kinetics, particle porosity, particle size, and flow rate on the breakthrough behaviour of the solute in an affinity packed column.

\section{Model Development}

The model developed incorporates the typical features of an affinity column packed with porous particles which is schematically represented in Fig. 1. The following basic assumptions are needed for the general rate model used in this study. The model is based on the isothermal sorption of a single solute in dispersed plug flow through a packed column of monodisperse porous particles. The bulk liquid has a solute concentration $C(z, t)$, with a superficial velocity $u_{0}$ through a bed of length $L$, and void fraction $\varepsilon$. The particles are spherical with radius $R$ and porosity $\beta$. The concentration of the solute adsorbed on the porous bead is $q_{\mathrm{i}}(r, z, t)$ and the solute concentration in the pores is $C_{\mathrm{i}}(r, z, t)$. It has been assumed that the effective diffusivity is independent of concentration, mass transfer to the surface of the adsorbent is governed by a film model [16] characterised by a mass transfer coefficient $k_{\mathrm{f}}$, and the immobilised ligand is distributed uniformly throughout the interior of the particle.

\subsection{Adsorption}

In the adsorption step, solute $(P)$ of interest is adsorbed onto the matrix on which the ligand $(L)$ is immobilised. The affinity interaction between the protein and vacant immobilised ligand is assumed to be of the form: ${ }^{1)}$

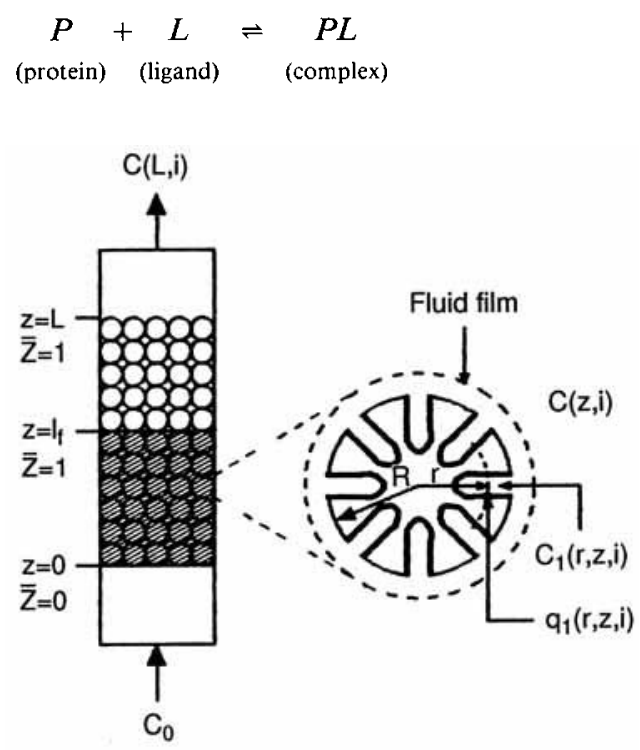

Fig. 1. Model of the affinity packed column.

1) List of symbols at the end of the paper. 
Monovalent adsorption is considered in the present study. Assuming that the affinity interaction is second order in the forward direction and first order in the reverse direction, the mass balance equation for solute adsorbed on solid phase can be written as:

$\frac{\partial q_{\mathrm{i}}}{\partial t}=k_{1} C_{\mathrm{i}}\left(Q_{\mathrm{m}}-q_{\mathrm{i}}\right)-k_{2} q_{\mathrm{i}}$

Eq. (1) simplifies to Langmuir's isotherm at equilibrium.

$q_{\mathrm{i}}=\frac{Q_{\mathrm{m}} K_{\mathrm{L}} C_{\mathrm{i}}}{\left(1+K_{\mathrm{L}} C_{\mathrm{i}}\right)}$

A mass balance over a section of the affinity packed column gives the following continuity relation:

$u_{0} \frac{\partial C}{\partial Z}+\varepsilon \frac{\partial C}{\partial t}+\left.\frac{3(1-\varepsilon)}{R} D_{\mathrm{i}} \frac{\partial C_{\mathrm{i}}}{\partial r}\right|_{\mathrm{r}=\mathrm{R}}=E_{\mathrm{z}} \frac{\partial^{2} C}{\partial Z^{2}}$

The various terms in the above equation account for convective transfer of the solute, accumulation in the interstitial spaces, solute uptake in the porous beads, and axial dispersion, respectively.

For a given porous particle, the following equation describes the diffusion of the solute into the pores with adsorption at the pore surface:

$D_{\mathrm{i}}\left(\frac{\partial^{2} C_{\mathrm{i}}}{\partial r^{2}}+\frac{2}{r} \frac{\partial C_{\mathrm{i}}}{\partial r}\right)-\frac{\partial C_{\mathrm{i}}}{\partial t}-\frac{(1-\beta)}{\beta} \frac{\partial q_{\mathrm{i}}}{\partial t}=0$

Assuming that there is no protein in the column in the beginning, the initial conditions can be written as follows. Initial conditions:

At $Z \geq 0, t=0$.

$C=0$

$C_{\mathrm{i}}=0$

$q_{\mathrm{i}}=0$

In order to include axial dispersion at the inlet of the column, and mixing at the exit of the column, the Danckwerts' [17] boundary conditions are used.

Boundary conditions:

For the column:

At $Z=0$

$\frac{u_{0} C_{0}}{\varepsilon}=\frac{u_{0} C}{\varepsilon}-E_{\mathrm{z}} \frac{\partial C}{\partial Z}$

At $Z=L$

$\frac{\partial C}{\partial Z}=0$
In general, axial dispersion should be considered at the entrance of the column because a steep first-order spatial gradient exists there. When axial dispersion is insignificant, i.e., $E_{z}$ approaches zero, Eq. (8) reduces to $C=C_{0}$ at the entrance, which is the case of a step input.

For the particle:

At $r=0$

$\frac{\partial C_{\mathrm{i}}}{\partial r}=0$

The concentrations $C_{\mathrm{i}}$ and $C$ in the pores of the particle and in the bulk liquid surrounding the particle respectively are coupled by the rate of mass transfer through the fluid film.

$$
\begin{aligned}
& \text { At } r=R \\
& \frac{k_{\mathrm{f}}}{\beta}\left(C-\left.C_{\mathrm{i}}\right|_{\mathrm{r}=\mathrm{R}}\right)=\left.D_{\mathrm{i}} \frac{\partial C_{\mathrm{i}}}{\partial r}\right|_{\mathrm{r}=\mathrm{R}}
\end{aligned}
$$

\section{Numerical Solution Strategy}

A moving front boundary value problem has to be proposed because of the low velocity and long column, as most parts of the column are not drenched at different times. The solution of the coupled non-linear second-order PDEs together with the boundary conditions requires computation by numerical methods. A moving finite element orthogonal collocation method is applied with respect to the space variables $(z$ and $r$ ) of the partial differential equations of the model to evaluate the breakthrough behaviour of the solute.

In order to apply collocation on a finite element [18], two concepts are utilised. The first one is the introduction of the width of an effective process zone, $z_{\mathrm{f}}$, where alone all the changes in the dependent variables occur. This zone moves with time until the entire column is drenched with the liquid. This concept is shown schematically in Fig. 1. The second one is the immobilisation of the moving boundary and collocation in the transformed coordinates. The front is immobilised by defining, $\bar{Z}=z^{*} / z_{\mathrm{f}}$, where $z^{*}=z / L$ and $z_{\mathrm{f}}=l_{\mathrm{f}} / L$. . Thus, at $z=0, \bar{Z}=0$ and at $z=l_{\mathrm{f}}, \bar{Z}=1$ and $C(z, t)$ is transformed to $C(\bar{Z}(z, t), t)$.

The above equations are non-dimensionalised by the following dimensionless variables:

$$
\begin{aligned}
& Y_{1}=q_{\mathrm{i}} / Q_{\mathrm{m}}, Y_{2}=C_{\mathrm{i}} / C_{0}, Y_{3}=C / C_{0} \\
& \bar{Z}=z^{*} / z_{\mathrm{f}}, R^{*}=r / R, T=t \times D_{\mathrm{i}} / R^{2}
\end{aligned}
$$

In the new coordinates, the mass balance Eqs (1)-(4) are transformed to 
$\frac{\partial Y_{1}}{\partial T}=H_{1} Y_{2}\left(1-Y_{1}\right)-H_{2} Y_{1}$

$Y_{1}=\frac{H_{3} Y_{2}}{\left(1+H_{3} Y_{2}\right)}$

$\frac{\partial Y_{3}}{\partial T}=\frac{H_{4}}{z_{\mathrm{f}}^{2}} \frac{\partial^{2} Y_{3}}{\partial \bar{Z}^{2}}+\left(\frac{\bar{Z}}{z_{\mathrm{f}}} \frac{\mathrm{d} z_{\mathrm{f}}}{\mathrm{d} T}-\frac{H_{5}}{z_{\mathrm{f}}}\right) \frac{\partial Y_{3}}{\partial \bar{Z}}-H_{6} H_{7}\left(Y_{3}-Y_{2}\right)$

$\frac{\partial Y_{2}}{\partial T}=\nabla^{2} Y_{2}-H_{8} \frac{\partial Y_{1}}{\partial T}$

In terms of dimensionless variables, the initial and boundary conditions (Eqs (5) through (11)) are as follows:

$Y_{3}=0$

$Y_{2}=0$

$Y_{1}=0$

$1=Y_{3}-\left(\frac{\varepsilon}{z_{\mathrm{f}} \mathrm{Pe}}\right) \frac{\partial Y_{3}}{\partial \bar{Z}}$

$\frac{\partial Y_{3}}{\partial \bar{Z}}=0$

$\frac{\partial Y_{2}}{\partial R^{*}}=0$

$\left.\frac{\partial Y_{2}}{\partial R^{*}}\right|_{\mathrm{R}^{*}=1}=H_{6}\left(Y_{3}-\left.Y_{2}\right|_{\mathrm{R}^{*}=1}\right)$

where constants $H_{1}$ through $H_{8}$ are defined as below:

$H_{1}=\frac{k_{1} R^{2} C_{0}}{D_{\mathrm{i}}}, \quad H_{2}=\frac{k_{2} R^{2}}{D_{\mathrm{i}}}$

$H_{3}=k_{\mathrm{L}} C_{0}, \quad H_{4}=\frac{E_{\mathrm{z}} R^{2}}{L^{2} D_{\mathrm{i}} \varepsilon}$

$H_{5}=\frac{u_{0} R^{2}}{D_{\mathrm{i}} L \varepsilon}, \quad H_{6}=\frac{k_{\mathrm{f}} R}{D_{\mathrm{i}} \beta}$

$H_{7}=\frac{3(1-\varepsilon)}{\varepsilon}, \quad H_{8}=\frac{(1-\beta) Q_{\mathrm{m}}}{\beta C_{0}}$

$\mathrm{Pe}=\frac{L u_{0}}{E_{\mathrm{z}}}$

The computer code for the governing mathematical equations is written in FORTRAN. The simulation is done by utilising NAG routine (D02EAF) on a Vax 88 system. This routine integrates a stiff system of first order ordinary differential equations, using a variable order variable step method implementing the backward differentiation formulae. Six interior points in the column and three interior points for the particle are used for the simulation studies.

\section{Results and Discussion}

Examples of simulations have been given to demonstrate the efficiency and robustness of a computer code based on a numerical procedure for the general rate model, which uses the finite element orthogonal collocation and Gear's stiff methods. The effect of axial dispersion, solute concentration, ligand loading, reaction kinetics, particle porosity, particle size, and flow rate on the breakthrough curve has been evaluated for a column packed with porous particles. The solute concentration profiles are evaluated with respect to time, i.e., in the form of a breakthrough curve. The base case parameter values used in the simulation are listed in Table 2 and are used throughout unless stated otherwise. Breakthrough time is defined as the time required for the exit solute concentration to reach $20 \%$ of that of inlet solute concentration $\left(C=0.2 C_{0}\right)$.

Before proceeding to the simulation of the detailed model, it is necessary to check the validity of the numerical code for the moving boundary collocation method. This is achieved by comparing the numerical results with an analytical solution for a simplified model given by Rasmuson and Neretnieks [19]. The analytical solution was obtained by assuming homogeneous diffusion in the particle phase and a linear equilibrium relation between free and adsorbed solute. Six interior points in the column and three interior points for the particle were used for the simulation. As seen from Fig. 2, the two results match perfectly well, confirming the validity of the numerical scheme.

Fig. 3 shows a typical breakthrough curve for the adsorption step. Much of the information needed to evaluate column performance is contained in this curve. Its shape is a result of a complex mix of equilibrium and non-equilibrium processes. The solute front takes $10 \mathrm{~min}$ (residence time) to reach the bottom of the column, and therefore, there is no fluid coming out before this time. The solution that comes

Table 2. Data for simulation

\begin{tabular}{llll}
\hline Parameter & This work & $\begin{array}{l}\text { Chase and } \\
\text { Horstmann [11] }\end{array}$ & $\begin{array}{l}\text { Boyer } \\
\text { and Hsu [13] }\end{array}$ \\
\hline$\beta(-)$ & 0.70 & 0.40 & - \\
$C_{0}\left(\mathrm{~g} \mathrm{~cm}^{-3}\right)$ & $1 \times 10^{-3}$ & $1.71 \times 10^{-3}$ & $1 \times 10^{-3}$ \\
$D_{\mathrm{i}}\left(\mathrm{cm}^{2} \mathrm{~s}^{-1}\right)$ & $3.2 \times 10^{-8}$ & $3.2 \times 10^{-8}$ & $3.33 \times 10^{-8}$ \\
$\varepsilon(-)$ & 0.40 & 0.40 & 0.40 \\
$E_{\mathrm{z}}\left(\mathrm{cm}^{2} \mathrm{~s}^{-1}\right)$ & $7.65 \times 10^{-1}$ & $1 \times 10^{-3}$ & $1 \times 10^{-3}$ \\
$k_{1}\left(\mathrm{~cm}^{3} \mathrm{~g}^{-1} \mathrm{~s}^{-1}\right)$ & 1.5 & 1.5 & $6.67 \times 10^{-2}$ \\
$k_{2}\left(\mathrm{~s}^{-1}\right)$ & $2.25 \times 10^{-5}$ & $2.25 \times 10^{-5}$ & $1.5 \times 10^{-5}$ \\
$k_{\mathrm{f}}\left(\mathrm{cm} \mathrm{s}^{-1}\right)$ & $2.5 \times 10^{-4}$ & $2.5 \times 10^{-4}$ & $2.833 \times 10^{-4}$ \\
$k_{\mathrm{L}}\left(\mathrm{cm}^{3} \mathrm{~g}^{-1}\right)$ & $6.67 \times 10^{4}$ & $6.67 \times 10^{4}$ & $4.45 \times 10^{3}$ \\
$L\left(\mathrm{~cm}^{3}\right)$ & 15 & 2.674 & 15 \\
$Q_{\mathrm{m}}\left(\mathrm{g} \mathrm{cm}^{-3}\right)$ & $30 \times 10^{-3}$ & $28.6 \times 10^{-3}$ & $61 \times 10^{-3}$ \\
$R(\mathrm{~cm})$ & $50 \times 10^{-4}$ & $45 \times 10^{-4}$ & - \\
$\left.u_{0}(\mathrm{~cm} \mathrm{~s})^{-1}\right)$ & $1 \times 10^{-2}$ & $8.5 \times 10^{-3}$ & $4.2 \times 10^{-3}$ \\
\hline
\end{tabular}




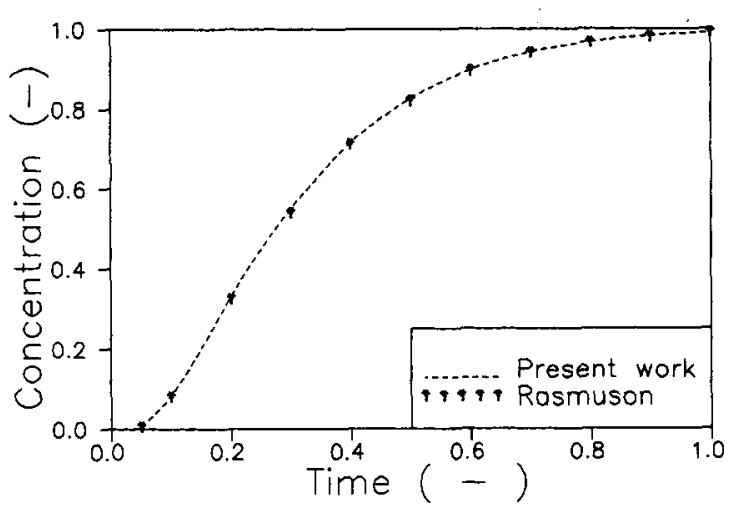

Fig. 2. Numerical comparison with analytical solution.

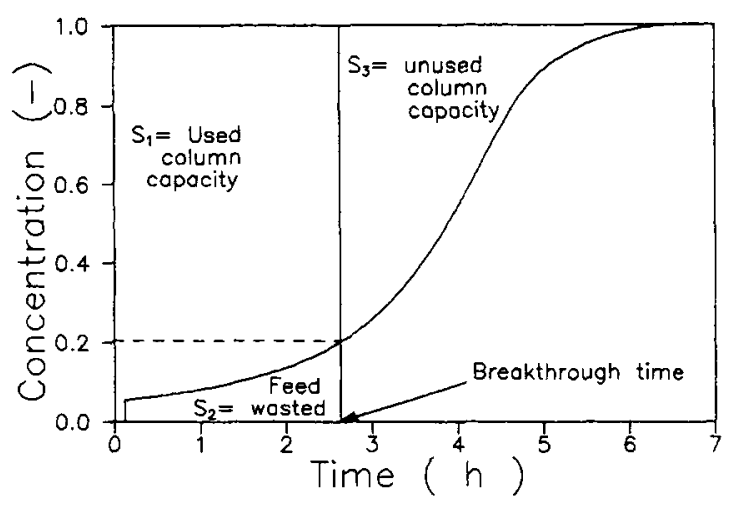

Fig. 3. Breakthrough curve for affinity adsorption.

out at 10 min contains unadsorbed solute at a concentration which depends on the capacity of the column. From $10 \mathrm{~min}$ onwards the effluent solute concentration increases with time. At very long times the column becomes saturated, and the effluent concentration equals the feed concentration. The maximum capacity of the column for a given inlet concentration is equal to the area behind the breakthrough curve. The amount of solute that remains in the effluent is, of course, the area under the curve. For quantitative comparison purposes, two efficiencies [20], i.e., solute recovery efficiency and bed utilisation efficiency, are defined in terms of area $S_{1}$ (= used column capacity), $S_{2}$ (= feed wasted), and $S_{3}$ (= unused column capacity) as shown in Fig. 3.

Solute recovery efficiency $=S_{1} /\left(S_{1}+S_{2}\right)$

Bed utilisation efficiency $=S_{1} /\left(S_{1}+S_{3}\right)$

The determination of the breakthrough point and the shape of the curve affects the efficiencies of adsorption. Solute recovery and bed utilisation efficiencies are calculated at a breakthrough concentration of $C=0.2 C_{0}$ for various parameters. Table 3 presents both the solute recovery and bed utilisation efficiencies for different parameters.

Chase and Horstmann [11] reported the experimental data for the affinity adsorption of immunoglobulin $\mathrm{G}$ onto Pro-
Table 3. Solute recovery efficiency and bed utilisation efficiency for various parameters

\begin{tabular}{lll}
\hline Parameter & $\begin{array}{l}\text { Solute recovery } \\
\text { efficiency }(\%)\end{array}$ & $\begin{array}{l}\text { Bed utilisation } \\
\text { efficiency }(\%)\end{array}$ \\
\hline $\mathrm{Pe}$ & & \\
0.20 & 90 & 70 \\
20.0 & 97.6 & 84.2 \\
$C_{0}$ & 90.9 & 65.4 \\
$5 \times 10^{-4}$ & 90 & 70 \\
$1 \times 10^{-3}$ & & \\
$Q_{\mathrm{m}}$ & 90 & 70 \\
$30 \times 10^{-3}$ & $\left.93^{\mathrm{a}}\right)$ & $\left.73.2^{\mathrm{a}}\right)$ \\
$60 \times 10^{-3}$ & 92.2 & 76.4 \\
$k_{1}$ & $\left.94.5^{\mathrm{a}}\right)$ & $\left.78.6^{\mathrm{a}}\right)$ \\
1.5 & & \\
& 90 & 70 \\
2 & $\left.92.5^{\mathrm{a}}\right)$ & $\left.73.1^{\mathrm{a}}\right)$ \\
$\beta$ & 92.6 & 72.5 \\
0.70 & $\left.93.2^{\mathrm{a}}\right)$ & $\left.74.7^{\mathrm{a}}\right)$ \\
0.74 & & 70 \\
$R$ & 90 & 58.7 \\
$5 \times 10^{-3}$ & 88.8 & 70 \\
$1 \times 10^{-2}$ & 90 & 31.5 \\
$u_{0}$ & 97.5 & 70 \\
$1 \times 10^{-2}$ & 90 & 81.2 \\
$4 \times 10^{-3}$ & 93.5 & \\
\hline & -60 &
\end{tabular}

a) Corresponding values for the Langmuir case.

tein A immobilised on agarose matrices. Fig. 4 shows the comparison between our model prediction and the experimental data of Chase and Horstmann [11]. Chase and Horstmann [11] modeled the above affinity system by neglecting axial dispersion. Our model differs from that of Chase in the inclusion of axial dispersion and Danckwerts' [17] boundary conditions. Chase and Horstmann [11] observed that the shape of the breakthrough curve is sensitive to the value of the dissociation constant $\left(K_{\mathrm{d}}\right)$. A particle porosity $(\beta)$ of 0.40 is used in our simulation. The model prediction compares well. The values of parameters used for prediction of the data of Chase and Horstmann [11] are given in Table 2 .

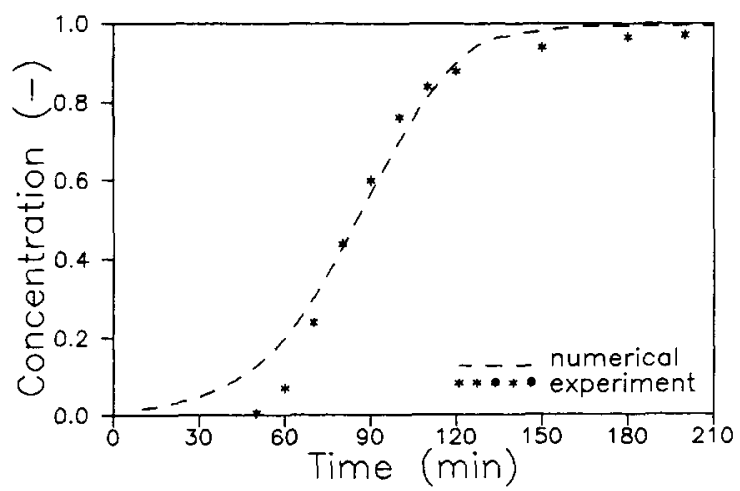

Fig. 4. Breakthrough curve comparison with experimental data. 


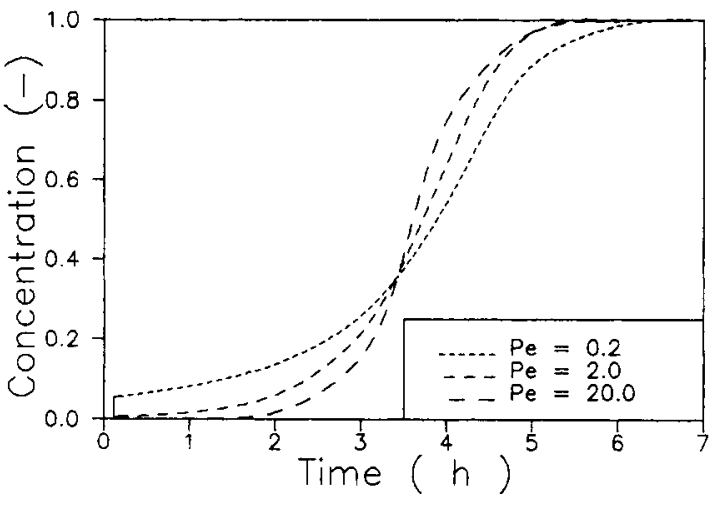

Fig. 5. Effect of axial dispersion on breakthrough curve.

Fig. 5 explains the effect of axial dispersion on the breakthrough curve. Axial dispersion affects the "sharpness" of the breakthrough curve. The velocity of the mobile fluid and the particle size distribution are the important physical factors that influence the dispersion in packed columns. Higher velocities and larger particles lead to more dispersion. At high dispersion, i.e., small Peclet number $\left(\mathrm{Pe}=L u_{0} / E_{\mathrm{z}}\right)$, the breakthrough curves are broadened, the time of total saturation is delayed and the loading capacity at the point of breakthrough is decreased. As dispersion decreases, i.e., Pe increases, the breakthrough curves become sharper. The higher the Peclet number, the lesser the axial dispersion, and hence the lesser the back mixing. This column operates with lower Peclet number, i.e., 0.2. The breakthrough curve for Peclet number 20.0 has better solute recovery and adsorbent utilisation efficiencies compared to that of the base case curve. Higher Peclet numbers are preferred for efficient operation.

Fig. 6 displays the effect of solute concentration on the breakthrough curve. The change in inlet solute concentration markedly affects the shape and position of the breakthrough curve. The higher the solute concentration, the faster the breakthrough. The results in Table 3 show that solute recovery efficiency decreases and adsorbent utilisation efficiency increases as $C_{0}$ increases. Lean feed is found to be better for solute recovery. However, the quantity of

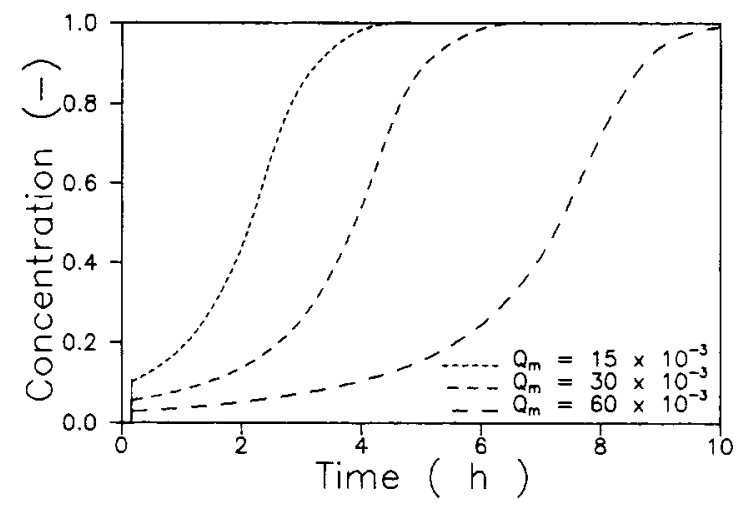

Fig. 7. Effect of ligand capacity on breakthrough curve. $Q_{\mathrm{m}}$ in $\mathrm{g} \mathrm{cm}^{-3}$.

solution to be supplied is large when the solute concentration is low and an optimum value has to be arrived at.

Fig. 7 depicts the effect of ligand capacity on the breakthrough curve. For the same inlet solute concentration, breakthrough curves are evaluated for three different ligand capacities. The higher ligand capacity leads to more uptake of solute and, therefore better bed utilisation. Langmuir's equilibrium adsorption isotherm was used for comparison in the model. The corresponding efficiencies for the Langmuir case are shown in Table 3 . It can be seen that as the loading capacity increases, the difference between kinetic and equilibrium (Langmuir) efficiencies decreases. At higher ligand loading, the Langmuir equilibrium is attained. Similar experimental observations have been reported by Boyer and Hsu [13] for adsorption of alcohol dehydrogenase on Cibacron Blue-Sepharose CL-6B adsorbents. The parameter values used in model simulation are tabulated in Table 2. Boyer and Hsu [13] modeled their experimental system by neglecting axial dispersion. There is no separate transport equation for the particle phase. The rate constants in the rate of adsorption equation are not the intrinsic rate constants, but are lumped parameters which reflect the contributions of mass transport as well.

In Fig. 8 the experimental breakthrough curves of Boyer and Hsu [13] for various bed capacities are compared with

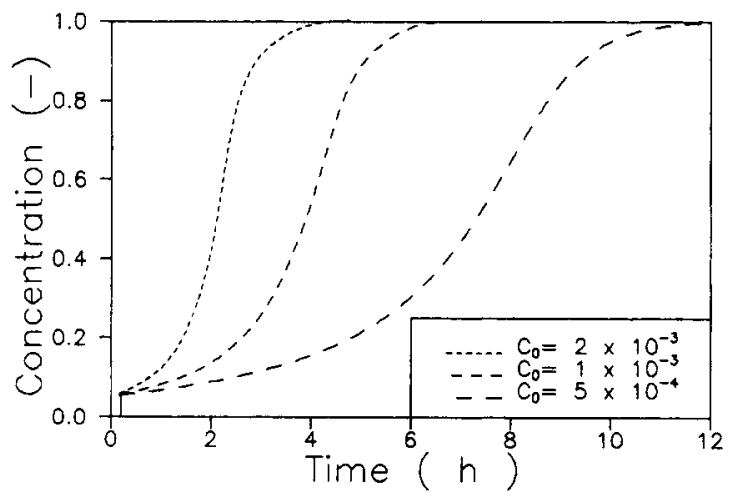

Fig. 6. Effect of solute concentration on breakthrough curve. $C_{0}$ in $\mathrm{g} \mathrm{cm}^{-3}$.

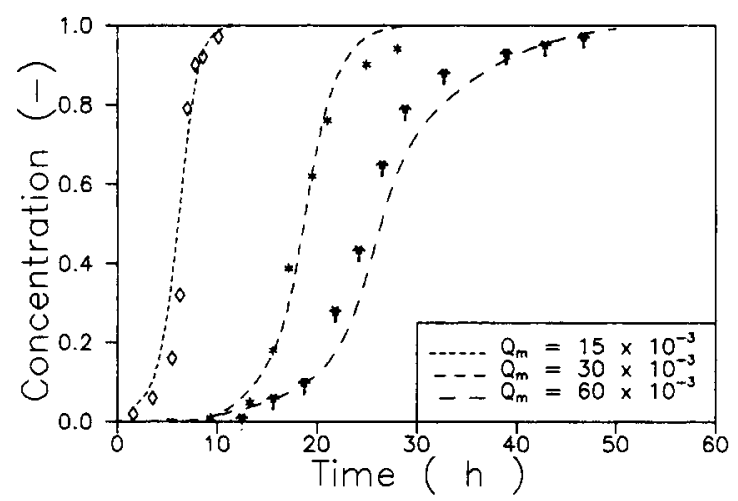

Fig. 8. Experimental breakthrough curves for different bed capacities. $Q_{\mathrm{m}}$ in $\mathrm{g} \mathrm{cm}^{-3}$. 
our model predictions. Higher bed capacities need longer saturation times. The multiple breakthrough times can be seen in Fig. 8, i.e., breakthrough times increase as ligand capacity increases.

Fig. 9 evaluates the effect of reaction kinetics on the breakthrough behaviour of the solute. The larger the forward rate constant, the sharper the breakthrough, thus improving adsorption efficiency. Higher values of the forward rate constant allow local equilibrium conditions to be approached more quickly. Kinetic rate constants can be changed by changing the $\mathrm{pH}$ of the medium. The comparison with Langmuir's isotherm case is made by substituting the corresponding $k_{\mathrm{L}}$ values in the model. It can be observed from Table 3 that, as the forward rate increases, the difference between kinetic and equilibrium (Langmuir) efficiencies decreases. At high forward rate constants, the Langmuir equilibrium is attained.

Fig. 10 examines the effect of particle porosity on breakthrough curve. The effective diffusion coefficient is directly proportional to the porosity of the particles. A small change in porosity has a pronounced effect on the breakthrough curve as it will affect the ligand capacity. Lower porosity leads to higher ligand capacity and vice versa. If the porosity is increased the breakthrough curve is shifted to the left. It can be observed from Table 3 that a small porosity has slightly better efficiencies.

Fig. 11 is the comparison of breakthrough curves for three particle sizes. Change in particle size affects the film mass transfer coefficient. For example, if $u_{0}$ is unchanged, the Wilson and Geankoplis correlation [21] indicates that $k_{\mathrm{f}}$ is proportional to $R^{-2 / 3}$. As reported in the literature, smaller size particles are found to be efficient. Breakthrough is faster for larger particle sizes.

Fig. 12 shows the effect of flow rate on the breakthrough behaviour of the protein. Flow rate affects the film mass transfer coefficient. If $R$ is unchanged, the Wilson and Geankoplis correlation [21] indicates that $k_{\mathrm{f}}$ is proportional to $u_{0}^{1 / 3}$. Breakthrough is faster for higher flow rates. When the flow rate is low a decrease in the spreading of the curve results in large solute recovery and bed utilisation efficiencies. High flow rates result in poor adsorption efficiencies. Low flow rates increase the time that the solute is in contact with the solid-phase, allowing more time for adsorption and permitting near-local equilibrium conditions.

\section{Conclusions}

Affinity chromatography is based on specific biological recognition and selective binding described by a nonlinear rate expression. In general, mass transfer effects, i.e., convective dispersion, fluid to particle mass transfer, and intraparticle diffusion will have a significant influence on the dynamics of the process. In the present study the following parameters have been shown to affect the breakthrough behaviour of an affinity packed column: axial dispersion,

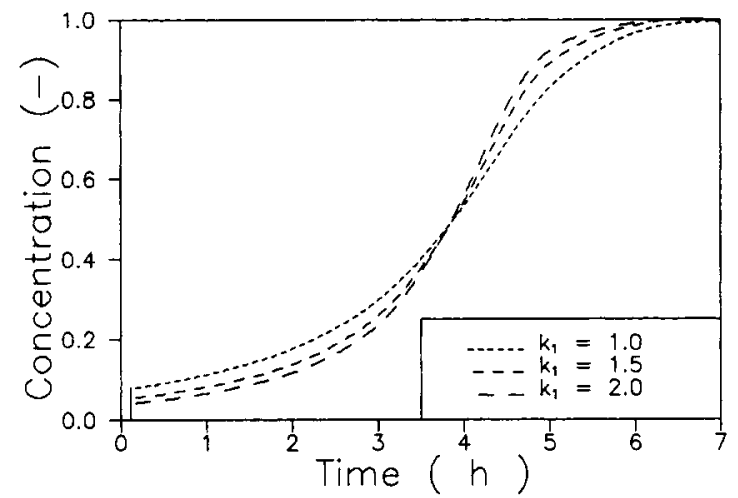

Fig. 9. Effect of reaction kinetics on breakthrough curve. $k_{1}$ in $\mathrm{cm}^{3}$ $\mathrm{g}^{-1} \mathrm{~s}^{-1}$.

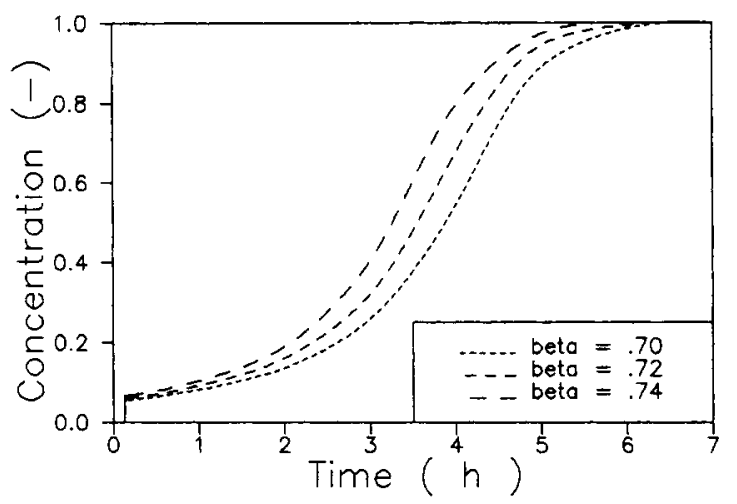

Fig. 10. Effect of particle porosity on breakthrough curve.

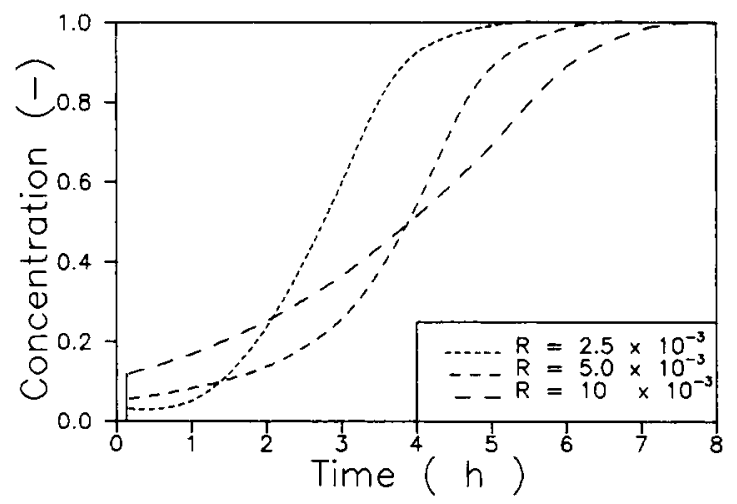

Fig. 11. Effect of particle size on breakthrough curve. $R$ in $\mathrm{cm}$.

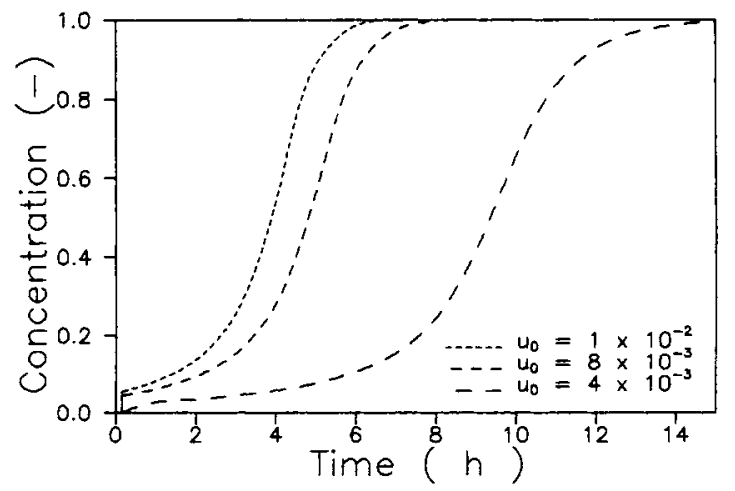

Fig. 12. Effect of flow rate on breakthrough curve. $u_{0}$ in $\mathrm{cm} \mathrm{s}^{-1}$. 
inlet solute concentration, ligand loading, surface reaction kinetics, particle porosity, particle size, and flow rate. The effect of axial dispersion is significant and affects both the solute recovery and adsorbent utilisation efficiencies. In this work, a general model is presented to describe monocomponent biospecific adsorption in a packed column. The model accounts for film and pore diffusion mass transfer resistances, axial dispersion, as well as the rate of interaction between solute and ligand. A robust and efficient numerical method has been used for the solution of the model. This method can be applied to a number of problems which exhibit steep moving profiles of the dependent variable. It represents a viable alternative to other established methods such as modified Crank-Nicolson, moving Galerkin finite element, etc. The model equations presented can be modified to account for linear, Freundlich, and Langmuir adsorption isotherms. A thorough experimental verification of the model equations presented in this work remains to be done. Although some researchers have reported breakthrough curves from affinity columns, most have not been accompanied by sufficient information such as bed length, flow rate, and particle size. Our model predictions for the experimental breakthrough curves compared well. Breakthrough curves are needed at different flow rates and concentrations. By using the same governing mathematical equations, but by changing the initial and boundary conditions, the elution step can be modeled. Experiments for purification of Concanavalin A on Sephadex beads are underway to verify the proposed model.

Received: September 23, 1993 [CET 600]

\section{Symbols used}

$\begin{array}{ll}C & {\left[\mathrm{~g} \mathrm{~cm}^{-3}\right]} \\ C_{\mathrm{i}} & {\left[\mathrm{g} \mathrm{cm}^{-3}\right]} \\ C_{0} & {\left[\mathrm{~g} \mathrm{~cm}^{-3}\right]} \\ D_{\mathrm{i}} & {\left[\mathrm{cm}^{2} \mathrm{~s}^{-1}\right]} \\ E_{\mathrm{z}} & {\left[\mathrm{cm}^{2} \mathrm{~s}^{-1}\right]} \\ K_{\mathrm{d}} & {\left[\mathrm{g} \mathrm{cm}^{-3}\right]} \\ k_{\mathrm{f}} & {\left[\mathrm{cm} \mathrm{s}^{-1}\right]} \\ k_{\mathrm{L}} & {\left[\mathrm{cm}^{3} \mathrm{~g}^{-1}\right]} \\ k_{1} & {\left[\mathrm{~cm}^{3} \mathrm{~g}^{-1} \mathrm{~s}^{-1}\right]} \\ k_{2} & {\left[\mathrm{~s}^{-1}\right]} \\ L & {[\mathrm{~cm}]} \\ l_{\mathrm{f}} & {[\mathrm{cm}]} \\ \mathrm{Pe} & \\ q_{\mathrm{i}} & {\left[\mathrm{g} \mathrm{cm}^{-3}\right]} \\ Q_{\mathrm{m}} & {\left[\mathrm{g} \mathrm{cm}{ }^{-3}\right]} \\ r & {[\mathrm{~cm}]} \\ R & {[\mathrm{~cm}]} \\ t & {[\mathrm{~s}]} \\ T & \\ u_{0} & {[\mathrm{~cm} \mathrm{~s}]}\end{array}$

$\begin{array}{ll}Y_{1} & \\ Y_{2} & \\ Y_{3} & \\ & \\ Z & {[\mathrm{~cm}]} \\ Z & \\ z_{\mathrm{f}} & \\ z^{*} & \end{array}$

\section{Greek symbols}

$\beta$
$\varepsilon$
$\nabla$

$$
\begin{aligned}
& \text { particle porosity } \\
& \text { column void fraction } \\
& \text { gradient }\left(\mathrm{cm}^{-1}\right)
\end{aligned}
$$

dimensionless local adsorbed solute concentration $\left(=q_{\mathrm{i}} / Q_{\mathrm{m}}\right)$ dimensionless pore liquid concentration $\left(=C_{\mathrm{i}} / C_{0}\right)$

dimensionless solute concentration at any axial position $\left(=C / C_{0}\right)$

axial position in the percolation column dimensionless axial coordinate dimensionless front position dimensionless axial coordinate

\section{References}

[1] Affinity Chromatography, Pharmacia Fine Chemicals, Uppsala 1979.

[2] Janson, J.C., Trends Biotechnol. 2 (1984) pp. 31-38.

[3] Scouten, W. H., Affinity Chromatography: Bioselective Adsorption on Inert Matrices, Wiley, New York 1981.

[4] Katoh, S., Kambayashi, T., Deguchi, R., Yoshida, F., Biotechnol. Bioeng 20 (1978) pp. $267-280$.

[5] Sportsman, J.R., Wilson, G.S., Anal. Chem. 52 (1980) pp. $2013-2018$.

[6] Chase, H.A., Chem. Eng Sci. 39 (1984) pp. $1099-1125$.

[7] Arnold, F.H., Blanch, H.W., J. Chromatogr. 355 (1986) pp. $13-27$.

[8] Arnold, F.H., Blanch, H.W., Wilke, C.R., Chem. Eng J. 30(2) (1985) B9-B24.

[9] Vermeulen, T., Klein, G., Hiester, N. K., Chemical Engineer's Handbook, R.H. Perry C.H. Chilton, (Eds.). McGraw-Hill, Inc., New York 1973.

[10] Arve, B. H., Liapis, A.I., AIChE J. 33 (1987) pp. 179-193.

[11] Horstmann, B. J., Chase, H.A., Chem. Eng Res. Des. 67 (1989) pp. $243-254$.

[12] Gonzalez-Patino, F., Catalan, J., Galan, M.A., Chem. Eng Sci. 48 (1993) pp. $1567-1573$.

[13] Boyer, P.M., Hsu, J.T., Chem. Eng. Sci. 47 (1992) pp. $241-251$

[14] Finlayson, B.A., Nonlinear Analysis in Chemical Engineering, McGraw-Hill, Inc., New York 1980.

[15] Villadsen, J., Michelsen, M. L., Solution of Differential Equation Models by Polynomial Approximation, Prentice-Hall, Englewood Cliffs, New Jersey 1978.

[16] Foo, S.C., Rice, R.G., AIChE J. $2 I$ (1975) pp. 1149-1158.

[17] Danckwerts, P.V., Chem. Eng Sci. 2 (1953) pp. $1-13$.

[18] Ramachandran, P.A., Dudukovic, M.P., Chem. Eng Sci. 39 (1984) pp. $1321-1324$.

[19] Rasmuson, A., Neretnieks, I., AIChE J. 26 (1980) pp. 686-690.

[20] Bo-Lun Yang, Motonobu Goto, Shigeo Goto, Colloids Surfaces 37 (1989) pp. $369-378$.

[21] Wilson, E. J., Geankoplis, C. J., Ind. Eng Chem. Fundam. 5 (1966) 9. 